\title{
EMERGING CHEMISTRY ON THE OIL OF THE FUTURE. REVIEW PAPER ON DAN NOCERA'S RESEARCH
}

\author{
Daniela Duca \\ Advanced Inorganic Chemistry, Lafayette College, Easton, PA 18042, USA \\ E-mail:feedana@yahoo.com \\ "When you bite a green leaf, you are chewing the photons of light"
}

/Dan Nocera/

\begin{abstract}
The paper is an overview of Daniel Nocera's work towards obtaining energy from the splitting of water. The review describes the challenge of storing high energy bonds and presents some of the important advances the researcher managed to accomplish.
\end{abstract}

Keywords: renewable energy, photocatalysis, porphyrin macrocycles, Rh bimetallic complexes

\section{Designing the Oil of the Future}

The main reason I became curious in Dan Nocera's work was his genuine and active interest in the "molecular chemistry of renewable energy" (1) as he himself calls it. Essentially, he is developing new ways of obtaining energy from the splitting of water, because of the large pressure between the forecast of energy usage and the energy availability for the future. He built up his research on the oil of the future on a natural model, i.e. that of plants. Plants are basically capturing the solar energy and storing it in a very interesting form. As Nocera explains in a web cast, "The Role of New Technology in a Sustainable Energy", plants take up water, that has low energy bonds, use the solar energy to break up the low energy bonds and form high energy bonds. The plant then stores these and releases the energy difference when it is needed by combining the hydrogen and oxygen back to forming the low energy bonds of water. So, "when you bite a green leaf, you are chewing the photons of light" (1).

One of the interesting questions he brought up during his talk was why plants do not make plastics and waxes or other types of oil instead of water. It must be that water is the most efficient reactant and product. Thus, Nocera decided that the oil of the future must be water. The challenge that Nocera come across when working with water was mainly the storage of the high-energy $\mathrm{H}-\mathrm{H}$ or O-O bonds. The papers that I chose for this review concentrate on dealing with this challenge.

\section{The Chemistry}

Think

One of the ways to deal with the challenge of storing the high energy bonds is by synthesizing metal complexes or macrocycles that would be stable enough to store them but sensitive enough to light in order to release them. There are a couple of issues with this such as the efficiency of the process, the impact of side products, and the reusability of the initial reactants and so on.

Nocera looked at nature one more time. He knew that water is regenerated at the end of the cycle, and can be reused in subsequent cycles. Therefore, he felt the need of designing a chain of reactions in which the complexes used in holding the high energy bonds will be regenerated at the end and could be subsequently reused. The reactions use only light energy, thus the chain takes the form of a photocycle. The advantages of photocycles include the need of less initial reactants and higher product yields, since the reactants will be used over and over again. This deals partly with the efficiency issue and the reusability of the initial reactants.

The one thing left to design are the reactants. Here, the issue of side product is taken into account. Also, the reactants or any of the chemical compounds used in the process should be stable, non-toxic, easy to handle and relatively easy to synthesize. As source of hydrogen, he considered the hydrogen halides. As source of oxygen, he considered water. As for the compounds used to store or hold the high energy bonds or to help in their formation, Nocera considered 3 types of photocatalysts with different ligands that would have 2 electrons available in their inventories. Thus, Nocera's group tried two-electron mixed valency electrons with stabilizing ligands for the $\mathrm{M}^{\mathrm{n}}-\mathrm{M}^{\mathrm{n}+2}$ construct, metal complexed porphyrinogens and bimetallic macrocycles.

Do

In a paper published in 2004, Nocera and his collaborates describe their research on oxygen and hydrogen photocatalysis using the bimetallic complexes, porphyrinogens and macrocycles. The idea of developing the twoelectron mixed valence bimetallic complexes comes from the need of two electrons in a two electron step reaction. As 
previously studied, the one-electron mixed valence complexes are used in one electron steps. Hydrogen, oxygen and water activation are, however, multielectron processes and thus the need for two-electron mixed complexes.

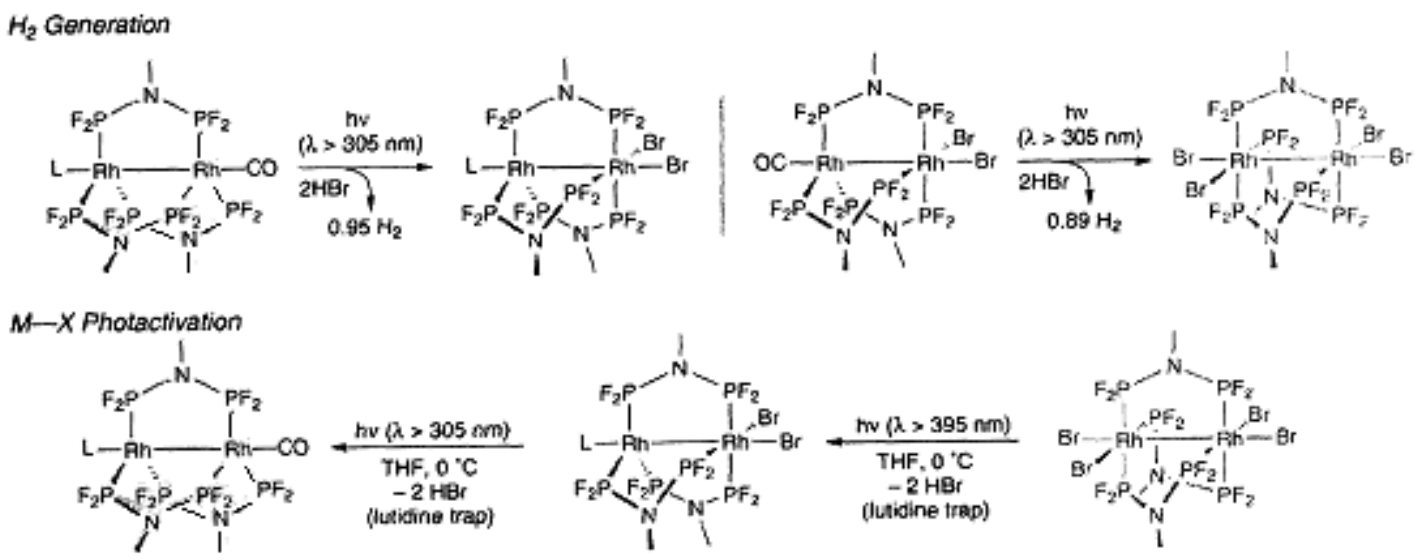

Fig.1. Reactions of the photocycle in Scheme 1 (2).

\section{Hydrogen}

Initially, the cycle was constructed around a homo two-metal complex with ligands that would easily leave the complex as soon as it reacts with hydrogen halides (Scheme 1). Two halogens would take the place of the ligand and a mole of $\mathrm{H} 2$ would be produced after photolysis generating a bimetallic halide blue intermediate that readily converts to a mixed valence bimetallic halide complex. By using a halogen trap and photoactivating the metalhalogen bond, the halogens can be replaced with the initial ligands and thus the complex would be regenerated (See Figure 1). For instance, when using $\mathrm{Rh}$ as the metal, it is necessary to have it complexed with ligands that stabilize the Rh0-RhII rather than RhI-RhI. The bidentate ligands bis(difluorophosphino)methylamine (dfpma) and bis(difluoroethoxyphosphino)methylamine (tfepma) (Figure 2) can destabilize the RhI-RhI and thus ease the conversion to the mixed valence complex. In addition, the CO ligand was studied for its ability to open a coordination site for the hydrogen halide attack (2).

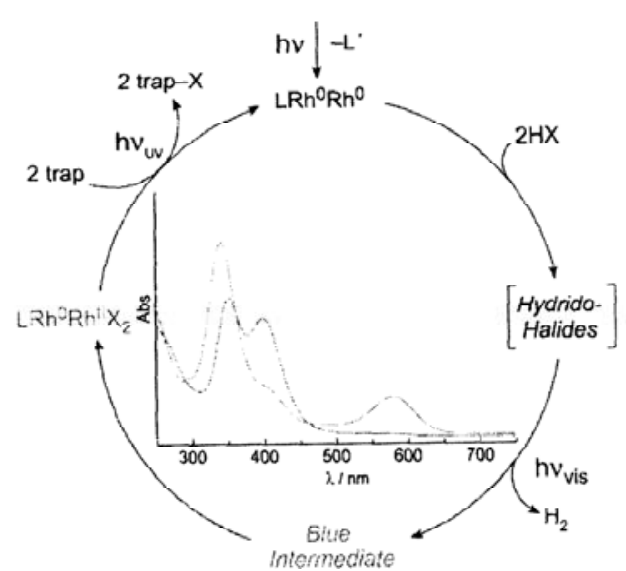

Scheme 1. Photocycle for Hydrogen production from hydrogen halides. (2)
More important however, would be complexes that are able to hold on to the hydrogen and then eliminate it as a high energy H-H bond. For that, iridium, a third-row metal, was used as the core metal of the complex, since it has a greater stability when bonded to $\mathrm{H}$, than $\mathrm{Rh}$, a second-row metal, does. So, except the increased stability that iridium provides to hydrogen and hydro-halides complexes, it also allows for an easy addition or elimination of the hydrogen. First, the hydrogen adds as an $\mathrm{H}-\mathrm{H}$ bond to the metal in the higher oxidation state. Next, one of the hydrogens can either pass to the second iridium to give rise to a fac stereochemistry, or, both hydrogens end up on the same iridium metal, thus forming the mer stereochemistry of the two electron mixed valence complex. The elimination of the hydrogen is just the reverse of the addition (2).

The importance of the partially reduced bimetallic complex intermediate being less stable than the $\mathrm{M}_{2}^{\mathrm{II}}$ complex is stressed. What is studied much more in detail or otherwise more clearly explained in this article is the relation between the ligands and the metal core, namely the metal-metal bond stabilization by different combinations of a bridging bidentate ligand. For instance, as shown in Figure 2, the ligand containing the electron donating nitrogen and two electron withdrawing phosphines on the sides suits best the purposes of the photocatalysts in the designed photocycle because it stabilizes the two-electron mixed valency bimetallic complex (3). 
Furthermore, experiments were carried out to determine the structure of the blue intermediate (See Scheme 1). First of all, it was determined that it is produced through an intra-molecular mechanism, which could happen only if the hydrogen adds to produce the mer stereochemistry. The blue intermediate is not stable in the presence of the electron donating nitrogen of the dfpm. In order to isolate and study it, however, Nocera's team replaced the dfpma ligands by the tfepm. Spectroscopic data showed that the intermediate was actually characterized by a d ${ }^{8}---d^{8}$ transition metal pattern of peaks. Thus, they synthesized Rh and $\mathrm{Ir}^{8}$ complexes where the metals with an oxidation of I are bonded to tfepm ligands and two halogens, i.e. $\mathrm{Cl}$. $\mathrm{Rh}_{2}{ }^{\mathrm{I}}$ was stable in solution, but $\mathrm{Ir}_{2}{ }_{2}^{\mathrm{I}}$ converts to the asymmetric mixed valence two electron complex $\mathrm{Ir}_{2}^{0, \mathrm{II}}$. Therefore, the hypothesis that predicts the tfepma and dfpma ligands to stabilize the mixed valence bimetallic complexes for their two $\pi$-accepting structures connected through a $\pi$-donating group holds. A new and more detailed cycle can thus be drawn (Scheme 2).

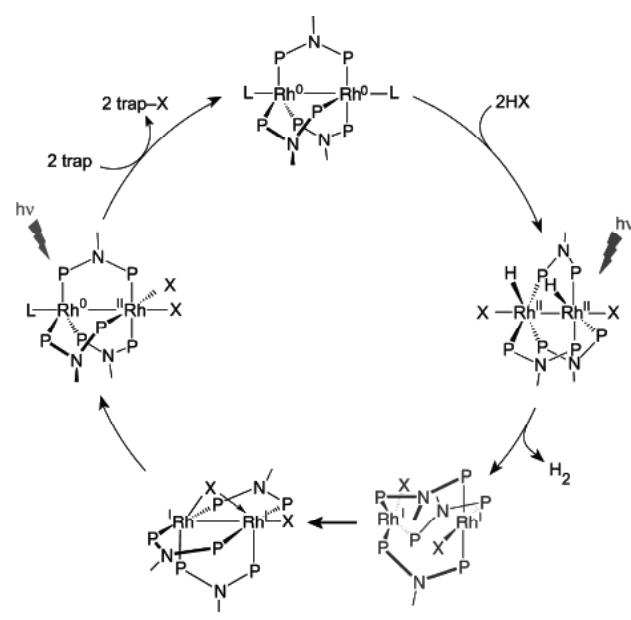

Scheme 2. Complete photocycle for hydrogen production from hydrogen halides using Rh complexes (3).

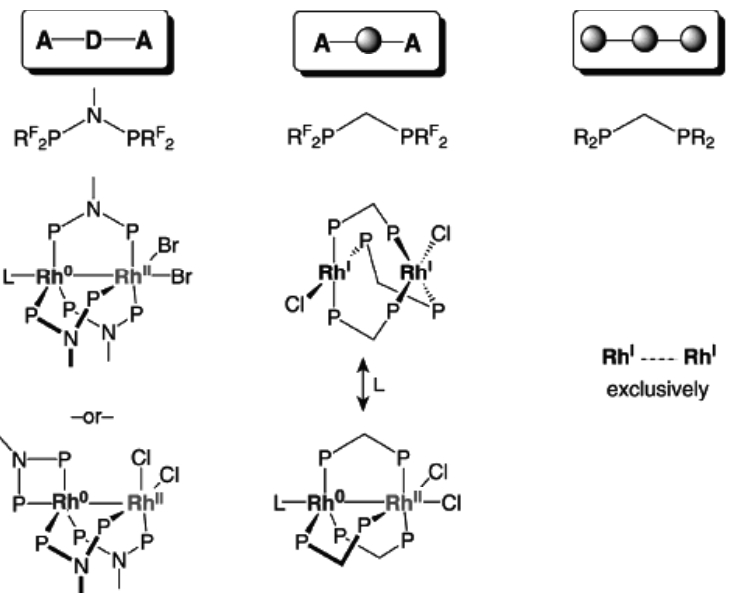

Fig. 2. The bimetallic Rh complexes stabilized by the bidentate ligands (3).

A crucial role in the photocycle is played by the metal halide complex. In order to close the cycle it is completely necessary to regenerate the initial compound from the metal halide complex. This is done by the elimination of the halogen. Nocera's team worked on increasing the efficiency of the halogen photo elimination by using early transition metals in high oxidation states for their larger oxidizing power(2). One option is then to use the complexes in which the early transition metals are most commonly encountered, such as porphyrins. Although porphyrin framework is favorable for halogen coupling, experiments proved activation of the M-X bond to fail as a result of the photoreduction of the porphyrin. Another option then would be to apply the design of the photocatalysts described earlier for Rh and Ir to early transition metals. The molybdenum and tungsten complexes developed in this manner did not work as well, for they were inert to oxidation (2).

The second type of complexes that Nocera's group developed are macrocycles. These are unique complexes and very different from the two electron mixed bimetallic ones because the ligands are the ones handling the electrons and not the metals as in the bimetallic complexes. Here they used porphyrinogens (Figure 3), but modified the core metal to $\mathrm{Zn}$ and used "redox-inert and spectroscopically silent counterions" (2). They studied the 3 oxidation states of the $\mathrm{Zn}$ porphyrinogen and compared them with the Rh and Ir bimetallic complexes. The distinction between the two lies in the fact that the intermediate of the bimetallic complex is symmetrically oxidized and reduced. In the case of the $\mathrm{Zn}$ porphyrinogen, the redox process occurs only at the margin of the complex, which does not affect the geometry of the metal. In the case of Rh and Ir, the oxidation state of one of the metals changes, and so does the geometry of the coordination sphere (2).

A subsequent idea for research thus emerged - develop catalysts for three electron processes. Nocera's group synthesized a porphyrinogen with an iron center that is supposed to be redox-active and thus change its oxidation state contributing one more electron to the process in addition to the two from the ligands (2).

At this point in time Nocera deduced that the only way that the energy efficiency of this cycle can be improved is by concentrating the research on the photoelimination step. In the earlier paper he tried out prophyrins and porphyrinogens as ligands which failed to ease the activation of the metal-halogen bond (2). Now he turns his attention to the third-row transition metal - gold. Some of the characteristics that favor gold as the core of the bimetallic complex are its multiple oxidation states, high reduction potential, good coordination geometry, better sensitivity to light when it comes to halogen photoelimination and, in addition, Au-Au complexes have already been studied (3).

A digold complex with tfepm and chlorine ligands was synthesized and structurally analyzed and compared to 
the previously developed Rh and Ir complexes. Also, a mixed metal complex Rh---Au was synthesized and structurally analyzed. It was determined that just like the $\mathrm{Au}_{2}$ complex, there is no formal bond between $\mathrm{Rh}$ and $\mathrm{Au}$ metals and that the geometry around the rhodium metal is almost square planar. In a more recent paper a follow-up of the idea of heterobimetallic mixed-valence complexes was investigated. This time the research concentrated on synthesizing a bimetallic complex that would subsequently produce a $\mathrm{Rh}^{\mathrm{II}}-\mathrm{Au}^{\mathrm{II}}$ complex. The latter could then be used in the photocycle (4).

The geometry around the two metals was determined by X-ray crystallography to be square planar for $\mathrm{Rh}^{\mathrm{I}}$ and distorted trigonal for $\mathrm{Au}^{\mathrm{I}}$. The distance between the two metals clearly attests that there is no formal bond. However, oxidation of this complex gives rise to a $\mathrm{Rh}^{\mathrm{II}}-\mathrm{Au}^{\mathrm{II}}$ complex that has formal bond with a pseudooctahedral geometry around the rhodium and a square planar geometry around the gold metal. Yet, $\mathrm{Rh}^{\mathrm{II}}-\mathrm{Au}^{\mathrm{II}}$ complex is not stable at ambient temperature and the bond between the two metals breaks, thus forming two other metal complexes: one of $\mathrm{Rh}^{\mathrm{III}}$ and another one of $\mathrm{Au}_{2}{ }^{\mathrm{I}}$. The conclusion drawn is that the bond in the heterobimetallic complex is highly polarized towards $\mathrm{Au}$, leading to a destabilization that induces one electron transfer from the rhodium's $\mathrm{d}^{7}$ to gold's $\mathrm{d}^{9}$ generating a $\mathrm{d}^{6}-\mathrm{d}^{10}$ complex. This heterobimetallic complex is therefore unstable toward the disproportionation of the bond and, subsequently, it cannot be used in the photocycle. That is why there is a need of investigating other metals that would be bonded so that there is no tendency towards electron transfers. Since the Au-X bond is sensitive to light and would contribute to the increase in quantum efficiency of the cycle, it would make sense to keep this metal and investigate one that would form a stable covalent bond with gold. Nocera is proposing $\mathrm{P}^{\mathrm{III}}$, which has a closer orbital energy to that of gold than rhodium (4).

\section{Oxygen}

When dealing with oxygen it is important to consider the complexity of making the high energy $\mathrm{O}-\mathrm{O}$ bonds and again look back at "Mother Nature", specifically at Photosystem II in plants. Just like with storing the $\mathrm{H}-\mathrm{H}$, there are some challenges with storing O-O. First, the source of oxygen could be water, which has to be activated in order to obtain the oxygen. This would definitely be a multi-electron process. Second, unlike for the hydrogen, complexes that would be holding the oxygen would probably be more complex geometrically since they have to accommodate a bigger atom. It is an extremely difficult task to keep in mind these two points in designing the chain of reactions that would activate oxygen from water, generate and store it for use, since all reactions in the chain should, in addition, be easy to control with close to no side products (3).

The complexes Nocera came up with for the oxygen photocatalysis are the Pacman porphyrins, which are basically 2 porphyrin macrocycles cofacially arranged and connected by xanthene or diphenyl furan. In his research during 2004, he initiated a light design of the oxygen photocycle. Unlike the hydrogen phtocatalysis in the oxygen photocycle, oxygen is not eliminated as a molecule, but rather taken in by the bisporphyrin complexes, which can eventually oxidize phosphines, sulfides and olefins, thus releasing the sought energy (2).

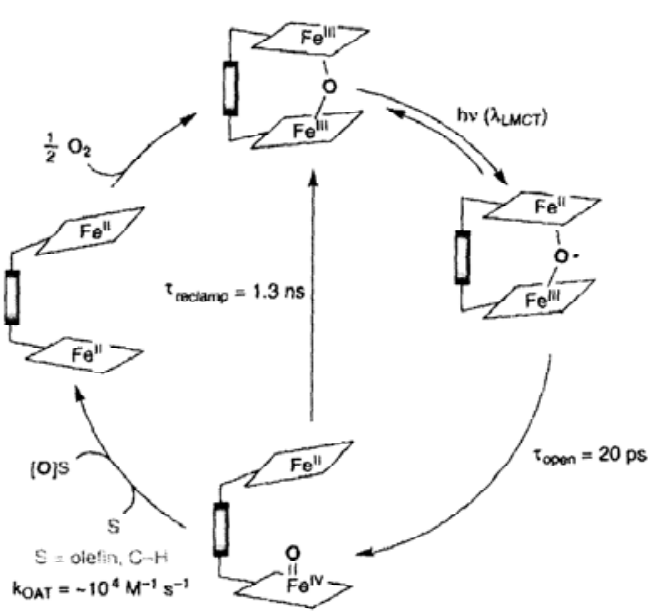

Scheme 3. Photocycle for the activation of oxygen with cofacial porphyrins bridged by xanthene or diphenyl furan (2).

As shown in Scheme 3, when oxygen is introduced in the system, the two irons immediately capture it. Light is then shined on the complex and one of the irons loses the covalent bond and returns to the original oxidation state, the other iron though undergoes an increase in the oxidation state becoming $\mathrm{Fe}^{\mathrm{IV}}$ with oxygen doubly bonded to it. This species then releases its oxygen in oxidizing phosphines and sulfides to regenerate the di-iron (II) bisphosphyrin (2).

The oxygen atom transfer efficiency depends on the bridge between the two porphyrin groups. Nocera's team determined that when using the diphenyl furan the complex is "spring-loaded", or in other words it has the ability to open up the space between the two porphyrins more than when xanthene is used in bridging. Thus, the diphenyl furan allows for more room where oxygen is attacked. To increase even more the oxidizing power of porphyrins some ligands were replaced with fluorine that is strong enough to leave the complex relatively deficient in electrons and thus more sensitive to photolysis. This modified complex is able to oxidize olephines in addition to sulfides and phosphines using a longer wavelength (2).

Nocera tried to increase even more the pocket size in order to avoid the formation of the bond between the metals and the oxygen. Iron has been replaced with $\mathrm{Co}(\mathrm{II})$ and xanthene and diphenylfuran bridging ligands were used again. These complexes are perfect for the reduction of oxygen to water, which is actually a 4 electron process. Still, they did not manage to prevent the formation of the $\mu$-oxo metal bond. The logical solution to avoid the formation of this bond was to synthesize a monomeric porphyrin complex and that is exactly what they did (Figure 3) (3). 

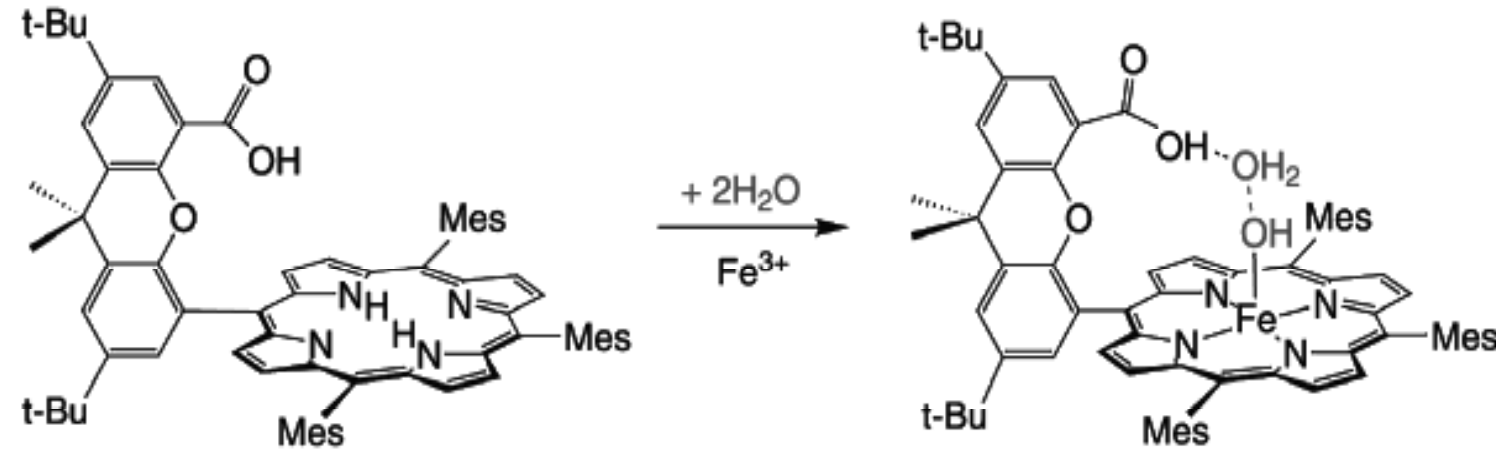

Fig. 3. The water hanging off of the pendant acid group on the Hangman platform (3).

This novel complex has a Hangman platform that permits the suspension of a water molecule over the metal hydroxide. From the structural point of view, the platform works in a closely similar manner to the cytochromes. It is basically an orthogonal proton coupled electron transfer process (PCET). So, while the oxygen undergoes a bond making reaction with the Fe of the complex, two electrons are taken up by the porphyrin ring, while the protons are transferred to the pendant acid group. It was determined that this group plays the same role as the water channels in natural enzymes. The reaction was monitored by stopped flow kinetics and it was found that the splitting of the $\mathrm{O}_{2}$ is clean and occurs heterolytically for the presence of the pendant acidic group (3).

The bisporhyrin complexes were developed and used in activating the oxygen through olephines, this latter mentioned monoporphyrin complex was developed to activate the oxygen from water by splitting it. As described in the paper (dampsey) the hydroxide ion is more difficult to oxidize so the research team worked on replacing the pending acid group which is unable to stabilize the ion with groups like amidine. In addition, they increased the electron withdrawing potential of the porphyrin macrocycle by fluorination, as it was also discussed in the previous paper. Further research that Nocera's group is looking into is the effects of photooxidants in the macrocycle and other macrocycles like Re(I) polypiridyl complexes (3).

\section{Get (or the All-in-a-snapshot-part)}

Through continuous and intense research Nocera and his team are on the way of developing the oil of the future and more specifically methods to get the energy out of it as efficiently as possible using only light as an input.

As specified throughout the paper, there are two main sides on which Nocera is concentrating: the hydrogen and the oxygen. Water contains low energy bonds and it is used by plants as a recyclable medium to create the high energy bonds of $\mathrm{H}-\mathrm{H}$ and O-O. Living organisms have very efficient mechanisms of obtaining and storing this high energy bonds and eventually using the energy off of them by producing water (1). Based on this idea, Nocera developed complexes that would activate hydrogen halides, olephins or water to obtain the high energy $\mathrm{H}-\mathrm{H}$ and O-O compounds. The complexes are usually metallic or bimetallic cores with stabilizing ligands (2-4).

With hydrogen, Nocera started out with a homobimetallic complex and a rough picture of the cycle that the complex would go through (2). He got as far as synthesizing a heterobimetallic complex with two electron mixed valency $\mathrm{Pt}^{\mathrm{III}}-\mathrm{Au}^{\mathrm{II}}$ and ligands dfpma and tfepma that stabilize the end product complex but not the symmetrically reduced and oxidized intermediate (4). The structure and properties of the intermediate where determined (3). The gold is used as one of the metals for its light sensitive $\mathrm{Au}-\mathrm{X}$ bond, fact that enhances the photoelimination to regenerate the starting complex, thus increasing the photochemical quantum yield (3). Pt will substitute the Rh in the M-Au complex, which was studied in one of the papers, but was found unfavorable for the process for the high metal-metal bond disproportionation, because of the large energy difference. Pt is still a d transition metal, but the energy gap between Pt and Au is smaller, hence the one electron transition from the $\mathrm{d}^{7}$ to the $\mathrm{d}^{9}$ would not be expected (4).

With oxygen, the approach is a little bit more difficult, since the final goal would be the splitting of water. Initially, Nocera sought the design of a similar photocycle as that for the hydrogen photooxidation, however the challenge was to accommodate the bigger oxygen. Therefore, he used a macrocycle made of two porphyrins bridged by xanthene or diphenyl furan instead (2). The aim was still the activation of water, but that was not possible for the formation of the bond between metal core of the porphyrin, i.e. Fe(III)-O-Fe(III) $(2,3)$. Although the energy efficiency of the cycle using this complex was not as best as it could be, the bisporhyrin could still be used for studying purposes. For instance, when shining light on it, the $\mathrm{Fe}^{\mathrm{II}} \mathrm{Fe}^{\mathrm{IV}}=\mathrm{O}$ complex forms. It reduces olefins easily, forming epoxides that are very reactive species. The initial bimetallic complex is then regenerated (2). The research was taken one step further synthesizing 
completely new monoporphyrin complexes as a Hangman platform that have the ability to hold an orthogonal PCET reaction for $\mathrm{O}-\mathrm{O}$ formation and heterolytic splitting with water being able to enter the complex and hang from the pending acid-base group (3).

\section{Breadth, Depth and the Rest}

One of the most interesting things I found about all three papers, is the assumption that the work on this research topic is followed by the reader starting the first article of the group published in this area. In other words, the more recent the article is the less information about the general goals of the research and the discoveries or conclusions made so far is expressed. For instance, the last article analyzed provides minimal data about the photocatalytic cycle and the purpose of it, whereas the first article (and probably more in one of the first ones published by Nocera's group) contains enough relevant information on the way in which the cycle works and how it has been developed. Probably, if I had time to venture, or if I knew exactly that reading another article, for that matter one of the first ones, would decrease my time spent on understanding the purposes behind Nocera's experiments, I would have definitely read one more.

A very strong point related to the writing style in the first paper were the small summaries or important points written in bullet form at the end of each compartment that described a certain part of the experiment. They reinforced the goal of the research as well as the actual importance of the experiments discussed antecedently.

\section{My Perspective and Proposal}

So far in this paper I described the research that Dan Nocera and his group developed in the field of molecular chemistry of renewable energy. I talked about water being the perfect reactant and product in this process. I also mentioned that the problem that scientists are facing at the moment is the lack of an efficient system to produce, hold and then release $\mathrm{H}_{2}$ and $\mathrm{O}_{2}$ to form water and give out energy. So, precisely this issue was at the core of Nocera's research. He approached it by constructing metal complexes that would hold the bonds and he designed reactions that would run in cycles and use solar energy. This system is, in essence, simulating the process of photosynthesis but outside the leaves. I will take my time in this last section, however, to propose some further investigations on Nocera's research, from my own perspective, that of an undergraduate. Hence, this section will focus on two different respects:

- improvements to Nocera's method of hydrogen production

- alternative complexes for oxygen activation.

Although the photocycle with the two-electron mixedvalence bimetallic complexes seems to be working, the questions that I posed myself from the start were related to the use of $\mathrm{HCl}$ as a reactant. How much hydrogen halide will be used? Where would this hydrogen halide come from? And what can be done with the halide obtained after the photoeleimination? Water is more abundant in comparison to hydrogen halides and would be perfect to use instead. I believe that the complex that Nocera developed, however, won't be able to hold the oxygen, and water might add to the metal

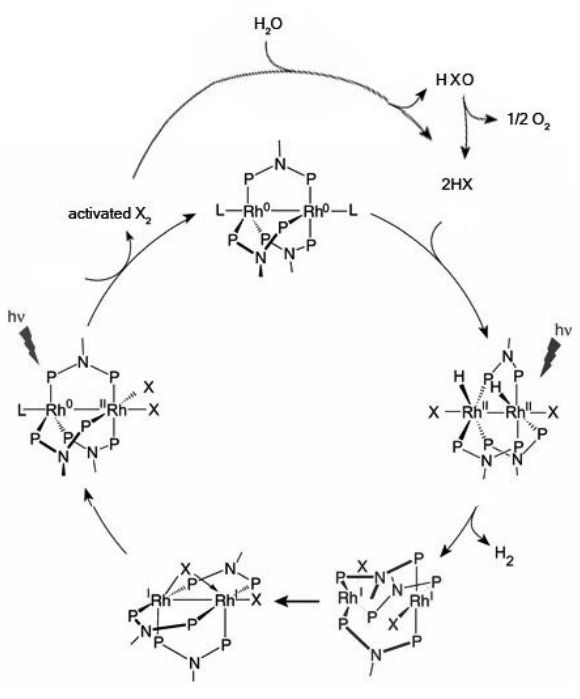

Fig. 4. Complete photocycle (from Ref. 2) with the incorporated water-halide cycle as aqua instead of separate hydrogen and oxygen molecules. With the hydrogen halides the whole hypothesis of producing energy from greener sources collapses, but water in this case is more difficult to handle. Hydrogen halides suit this photocycle better because although each of the hydrogen halide adds to one metal, the network of ligands and the two-electron mixed valency of the metals allow bridging transfers to occur and, hence, facilitate the photoelimination (3).

This whole process has been proven to work only on a laboratory level. If, however, it is upscaled Nocera would have to confront the hydrogen halide issue. In his research he determined that it is the photoactivation of the M-X bond that blocks the efficiency of the cycle. So, he changed the metal from rhodium to gold, which is known for its photosensitive halide bonding $(2,4)$. He also changed the solvent from 2,3-dimethyl-1,3-butadiene (DMBD) to tetrahydrofuran (THF). A ten fold increase in efficiency was observed (5). However, THF probably creates a problem, because it constitutes the halide radical trap and if the process is upscaled there will be some THF residue.

To increase the efficiency of the use of hydrogen halides and to avoid any THF residue a cycle that would 
regenerate the HX could be constructed (See Figure 4). For instance, if we use hydrogen chlorides we could try to regenerate $\mathrm{Cl}_{2}$ at the end of the photocycle according to this simplified reaction:

$2 \mathrm{HCl} \rightarrow \mathrm{H}_{2}+\mathrm{Cl}_{2}$

The eliminated chlorine could be activated and let to interact with water and produce the following $(7,8,10)$ :

$\mathrm{Cl}_{2}+\mathrm{H}_{2} \mathrm{O} \Leftrightarrow \mathrm{HCl}+\mathrm{HClO}$

It was determined that the equilibrium for the chlorine hydrolysis lies much more to the right than for bromine (8). Therefore, the use of chlorine as the halogen in the photocycle is more suitable. The $\mathrm{HCl}$ will be used in further reactions in the photocycle. The $\mathrm{HClO}$ under specific conditions could dissociate to form another mole of $\mathrm{HCl}$ and half the molar amount of oxygen (personal assumption, not found supporting literature):

$\mathrm{HClO} \rightarrow \mathrm{HCl}+1 / 2 \mathrm{O}_{2}$ by Nocera.

The oxygen product from this reaction could subsequently be activated by the oxygen photocycle also described

The reactions above can be integrated into Nocera's photocycle as depicted in Figure 4. The main focus of the cycle would be the photoelimination of the halogen without the use of a trap. The idea is to eliminate an activated halogen that would be able to interact with water and regenerate the hydrogen halide. The radical halogens formed during photolysis are very reactive species and could be used. As described earlier, in Nocera's experiments the radicals interact with the THF solvent $(2,3,5)$. We want them however to interact with the water.

One potential solution to this problem would be to apply a biphasic photoactivation ${ }^{1}$. In his early experiments, Nocera used DMBD as a solvent (2). DMBD is insoluble in water (6) and the bimetallic complex was reported to be stable in this solvent (2). The objective is to do a one-pot photoreaction with the complex sitting in DMBD, but have water present at the same time. As soon as light is shined, the M-X bond will be activated and halogen radicals will be formed. The photoelimination of the M-X bond is in the presence DMBD solvent as reported in Nocera's work as well, but the radicals would be able to move in the water phase. Theoretically, water could play the role of a trap for the radical, akin the THF. Since the DMBD solvent cannot serve as a trap for the halogen, as long as the surface contact with water is large enough, the halogen radical would recombine with itself and interact with water to generate $\mathrm{HCl}$ and $\mathrm{HClO}$ in a clean reaction.

Another potential solution could be redesigning the complex. Figure 5 presents a model of a theoretical complex. I used only two chelate ligands and I simplified them to the form shown in Figure 5, where they don't contain the methyl group off of the nitrogen and the fluorinated groups off of the phosphorous. The main goal here is to be able to dissolve the bimetallic complex in water and therefore achieve the photoelimination with water being the radical trap in the immediate vicinity. In addition, I simplified the structure of the complex to this point in order to be able to attack the two metal chores with 4 hydrogen halides instead of two. In this fashion, each cycle will give twice as much hydrogen, and the halide can then be recollected as hydrogen halide and reused in another cycle. The ligands used in addition to the bidentate ligands should not interfere with the solubility of the complex in water, for instance even halogens could be attached.

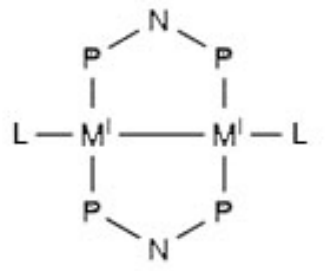

Fig. 5. Model bimetallic complex. $\mathrm{M}$ is the metal $\mathrm{Rh}$,Ir or $\mathrm{Au}$, and L stands for a ligand

The stability of the bimetallic complex from Figure 5 in water can be predicted by computational analysis as well as by carrying out the first few reactions in the cycle. In the work done by Nocera with the rhodium complex, one of the intermediates was blue in color and it was determined to be the $\mathrm{Rh}^{\mathrm{I}}-\mathrm{Rh}^{\mathrm{I}}$ complex. This intermediate would immediately disproportionate to $\mathrm{Rh}^{0}-\mathrm{Rh}^{\mathrm{II}}$, the bimetallic complex that would allow for the elimination of the halogen. It is the stability of the latter that is essential. Hence, it is important to check for the color change for the newly designed complex. This will prove the stability of the two mixed electron valence rhodium complex in water. In addition, the reaction could be monitored by ${ }^{103} \mathrm{Rh}$ NMR (11) with bulkier phosphorous substituents that slow down the reactions and allow for the isolation and purification of the intermediates (2).

In the water-hydrogen halide cycle that I developed in addition to the complete photocycle for the production of hydrogen (Figure 4), one of the side products obtained was HXO. The oxohydrogen halide can dissociate into $\mathrm{HX}$ and oxygen upon imposing alkaline conditions. There will then be a tendency to produce $\mathrm{H}^{+}$to counteract the basic environment. It has been reported in literature that hypobromous acid would disproportionate to produce $\mathrm{HBrO}_{3}$ and $\mathrm{HBr}(9)$. If this issue cannot be avoided by increasing the temperature to decompose $\mathrm{HBrO}_{3}$, then chlorine again turns out to be the better candidate because it does not disproportionate $(7,10)$. The oxygen obtained could be further activated through the porphyrin photocylcle also proposed by Nocera $(2,3)$.

A third possibility, and probably the easier one to be realized would be to apply the system developed for oxygen photoactivation to hydrogen. In this case the experiments will focus on characterizing the structure of the

\footnotetext{
${ }^{1}$ Dr. Nataro's suggestions
} 
metal porphyrinogen hydride complex and the reaction stoichiometry. At the same time porphyrinogen complexes of a range of metals like $\mathrm{Zn}, \mathrm{Fe}$ and Co should be tested for efficiency to eliminate the hydrogen. Similar to the oxygen photoactivation, complexes containing cofacial porphyrinogens and porphyrinogen with a Hangman complex hanging from the bridging xanthenes or diphenylfuran could be tested. I hypothesize that in the case of the bisporphyrinogens the hydrogen and the halide will add to the two different metals. The xanthene will reduce the space between the two porphyrinogens allowing for bridging transfer reactions to occur that will facilitate the hydrogen and chlorine elimination. One of the challenges encountered could be that during the halogen photoelimination, the halogen radical might abstract or remove the hydrogens off of the ligands.

In summary, the improvements I proposed for the hydrogen production cycle that Nocera developed are of two kinds and stem from the issues I believe Nocera might deal with when upscaling his energy production. First of all, I suggested an additional cycle to eliminate the possibility of side products and reactants. This additional cycle involves the usage of the photoeliminated halogen to regenerate the hydrogen halide. I hypothesized that a biphasic photoactivation would eliminate the problem of the halogen interacting with the solvent and creating solvent residues. Furthermore, I designed an alternative complex that would use water as a solvent and use it right away in the regeneration of HX. Lastly, the oxygen photocycle could be tested for hydrogen with different metals and ligand arrangements. Provided an abundant and clean renewable energy source is one of the main focuses of the development in science and technology and since hydrogen is probably the most ideal carrier, this research would be important for Nocera's photocatalysis since it deals with upscaling issues of the process.

\section{References}

[1]. "The Role of new Technology in a Sustainable Energy Economy" (2007). Webcast retrieved from http://mitworld. mit.edu/video/414/ on September 25, 2007.

[2]. Rosenthal J., Bachman J., Dempsey J. L., Esswein A. J., Gray T. G., Hodgkiss J. M., Manke D. R., Luckett T. D., Pistorio B. J., Veige A. S., Nocera D. G. (2005) Oxygen and hydrogen photocatalysis by two-electron mixedvalence coordination compounds. Coord. Chem. Rev. 249, 1316-1326

[3]. Dempsey J.L., Esswein A.J., Manke D.R., Rosenthal J., Soper J.D., Nocera D.G. (2005) Molecular chemistry of consequence to renewable energy. Inorg. Chem. 44, 6879-6892

[4]. Esswein A.J., Dempsey J.L., Nocera D.G. (2007) A $\mathrm{Rh}^{\mathrm{II}}-\mathrm{Au}^{\mathrm{II}}$ bimetallic core with a direct metal-metal bond. Inorg. Chem. 46, 2362-2364

[5]. Nocera D. excerpts from "Results from prior NSF support". Received on Nov. $20^{\text {th }}, 2007$.

[6]. Durrans T.H. (1971) Solvents 8e. Chapman and Hall: London.

[7]. Morris J.C. (1946) The mechanism of the hydrolysis of chlorine. J. Am. Chem. Soc. 68, 1692-1694

[8]. Lifshitz A., Perlmutter-Hayman B. (1960) The Kinetics of chlorine. I. Reinvestigation of the hydrolysis in pure water. J. Phys. Chem. 64 (11), 1663-1665

[9]. Beckwith R.C., Xiang Wang T., Margerum D.W. (1996) Equilibrium and kinetics of bromine hydrolysis. Inorg. Chem. 35, 995-1000

[10]. [Wang T.X., Margerum D.W. (1994) Kinetics of reversible chlorine hydrolysis. Temperature dependence and general acid/base assisted mechanism. Inorg. Chem. 33, 1050-1055

[11]. Sharpe A., Housecroft C.E. (2001) Inorganic chemistry 2e.Prentice Hall: Pearson Education. 